\title{
Prevention of sudden cardiac death in athletes: new data and modern perspectives confront challenges in the 21 st century
}

\author{
Jonathan Drezner, Babette Pluim, Lars Engebretsen
}

The sudden death of a young athlete on the playing field remains the most devastating medical event in sports. The tragic impact of these events compels sports medicine professionals and sport governing bodies to develop and implement effective preventive strategies. In this issue, the British Journal of Sports Medicine and the International Olympic Committee (IOC) partner to present the most current information relevant to sudden cardiac death (SCD) in athletes. First in a series of quarterly BJSM-IOC issues devoted to injury prevention and health protection in athletes, this issue is dedicated to preventing the worst injury of all: sudden death.

The objective of this issue is to highlight cardiovascular diseases in athletes through a compilation of commissioned reviews and original investigations authored by international experts in sports cardiology and sports medicine. The issue provides modern perspectives and recent data in the areas of epidemiology, diagnosis, screening and management.

Prevention of SCD in athletes remains one of the most debated topics in sports medicine. What is the most appropriate strategy to screen athletes for underlying cardiovascular disease? Does the presence of automated external defibrillators (AEDs) at athletic events effectively treat young athletes who experience sudden cardiac arrest (SCA)? The complexities surrounding these questions are addressed in several reports presented in this issue.

The screening debate is centred on the inclusion (or not) of a resting 12-lead electrocardiogram (ECG) in addition to a medical history and physical examination during the preparticipation athletic evaluation. At times, however, this global debate has stalled because of a continuing

Correspondence to: Jonathan Drezner, University of Washington, Seattle, Washington, USA; jdrezner@ fammed.washington.edu disagreement on the incidence of SCD in young athletes.

\section{INCIDENCE OF SCD IN ATHLETES: ESTIMATES VARY 10-FOLD!}

SCD is the leading cause of death in young athletes. ${ }^{1}$ However, the exact incidence of SCD is unknown, and it is difficult to compare incidence studies with profoundly different methodology and from widely different geographic locations. Estimates from the US range from 1:160 000 to 1:300 000 competitive athlete deaths per year due to cardiovascular diseases. ${ }^{12}$ These studies, while rigorous in effort, rely primarily on search of public media reports and other electronic databases and are at risk of underestimating the incidence of SCD due to the lack of a mandatory reporting system. In contrast, the Veneto region of Italy utilises a regional registry for juvenile sudden death and reported a SCD incidence of 1:28 000 for young competitive athletes (ages 12-35 years) prior to implementing a national screening program. ${ }^{3}$ Similarly, a recent prospective populationbased study conducted at 11 US and Canadian cities found an incidence of SCA from cardiovascular disease of 1:27 000 in children and young adults (ages 14-24). ${ }^{4}$ Thus, initial reports may have vastly underestimated the magnitude of the problem of SCD in athletes.

It is essential that the sports medicine community clarifies the purpose of cardiovascular screening in athletes. Is the goal of screening simply to prevent SCD? Or is the goal of screening to identify children at risk with cardiovascular disorders that can be managed via medical intervention and activity modification to reduce their risk of sudden death? The American College of Cardiology contends that the ultimate objective of preparticipation screening of athletes is the detection of "silent" cardiovascular abnormalities that can lead to SCD. ${ }^{5}$ From a primary screening perspective, perhaps it is the prevalence of cardiovascular conditions with the potential for sudden death that should heavily guide our screening procedures. The combined disease prevalence of all cardiovascular disorders that predispose young athletes to SCD is estimated at $0.3 \%(1: 333){ }^{6}$ In contrast to the disparities surrounding SCD incidence, this prevalence has been confirmed in many studies using ECG screening where the true positive rate for identified cardiovascular diseases ranges from $0.2 \%$ to $0.4 \%{ }^{3}{ }^{78}$

When evaluating the potential value or limitation of including ECG in a screening protocol, it is critical to recognise that the total positive and false positive rates for any ECG screening study or practice is immensely affected by the criteria chosen to define "abnormal." In this issue Corrado and colleagues ${ }^{9}$ present a comprehensive review on the interpretation of the ECG in athletes. Johnson and Ackerman ${ }^{10}$ also provide a persuasive report on the OTc interval, and Maron ${ }^{11}$ summarises methods to distinguish physiological from pathological hypertrophy in athletes. These references provide a modern framework to improve screening procedures in young athletes.

\section{TIME TO UPDATE ECG NORMS?}

There is an urgent need for uniform terminology when describing ECG findings in athletes. Many ECG changes once referred to as "abnormal" are now recognised as physiological and part of benign cardiac adaptation in athletes. Doctors interpreting ECGs in athletes should be familiar with common training-related ECG alterations that are normal variants. In contrast, training-unrelated ECG changes suggest the possibility of underlying pathology, require further diagnostic investigation and should be considered abnormal. In this issue, Papadakis and Sharma ${ }^{12}$ suggest that applying modern, strict ECG criteria to screen athletes can result in a low and acceptable false positive rate.

Although the debate over different screening strategies remains impassioned, it is important to appreciate that the international sports medicine community has more common purpose than it does dissent with respect to preventing SCD. There is universal agreement that SCD is catastrophic, athletes are at increased risk, effective prevention is critical and screening for occult disease is important. And, even though the precise frequency of SCD in athletes remains disputed, there is general agreement that vigorous exercise is a trigger for SCD in athletes with underlying cardiac disease. 
Many challenges remain regarding SCD prevention that must be addressed through further education and research. Unfortunately, even past screening recommendations that are universally accepted, such as the use of a comprehensive personal and family questionnaire to guide the preparticipation evaluation, have not been widely adopted. Doctors conducting these evaluations should be aware of the warning symptoms and family history that may indicate the presence of a lethal cardiovascular abnormality. In addition, to effectively implement ECG screening, complex issues regarding infrastructure, cost, feasibility and doctor education must be addressed through further study. To confront these challenges we must move beyond a debate grounded in incidence estimates and false positive rates that derive from studies with vastly different methodology and terminology. While the horizon for prevention of SCD looks promising, significant work still lies ahead.

BJSM and the IOC, partners in promoting the health of sportspeople the world over, look forward to your responses via the BJSM blog, (http://blogs.bmj.com/ bjsm), emails, or manuscript submissions. And please note that the IOC World Conference on Prevention of Injury and Illness in Sport, (http://www.ioc-preventionconference.org) in Monaco, 7-9 April 2011, will have SCD as one of its focus areas.

Competing interests: None declared.

Br J Sports Med 2009;43:625-626.

doi:10.1136/bjsm.2009.064592

\section{REFERENCES}

1. Maron BJ, Doerer JJ, Haas TS, et al. Sudden deaths in young competitive athletes: analysis of 1866 deaths in the United States, 1980-2006. Circulation 2009;119:1085-92.

2. Van Camp SP, Bloor CM, Mueller FO, et al. Nontraumatic sports death in high school and college athletes. Med Sci Sports Exerc 1995;27:641-7.

3. Corrado D, Basso C, Pavei A, et al. Trends in sudden cardiovascular death in young competitive athletes after implementation of a preparticipation screening program. JAMA 2006;296:1593-601.

4. Atkins DL, Everson-Stewart S, Sears GK, et al. Epidemiology and outcomes from out-of-hospital cardiac arrest in children: the Resuscitation Outcomes Consortium Epistry-Cardiac Arrest. Circulation 2009;119:1484-91.
5. Maron BJ, Zipes DP. 36th Bethesda Conference: eligibility recommendations for competitive athletes with cardiovascular abnormalities. J Am Coll Cardiol 2005; 45:1312-77.

6. Maron BJ, Thompson PD, Ackerman MJ, et al. Recommendations and considerations related to preparticipation screening for cardiovascular abnormalities in competitive athletes: 2007 update: a scientific statement from the American Heart Association Council on Nutrition, Physical Activity, and Metabolism: endorsed by the American College of Cardiology Foundation. Circulation 2007;115:1643-55.

7. Fuller CM, McNulty CM, Spring DA, et al. Prospective screening of 5,615 high school athletes for risk of sudden cardiac death. Med Sci Sports Exerc 1997:29:1131-8.

8. Wilson MG, Basavarajaiah S, Whyte GP, et al. Efficacy of personal symptom and family history questionnaires when screening for inherited cardiac pathologies: the role of electrocardiography. Br J Sports Med 2008;42:207-11.

9. Corrado D, Biffi A, Basso C, et al. 12-lead ECG in the athlete: physiological versus pathological abnormalities. Br J Sports Med 2009;43:669-76.

10. Johnson JN, Ackerman MJ. OTC: how long is too long? Br J Sports Med 2009;43:657-62.

11. Maron BJ. Distinguishing hypertrophic cardiomyopathy from athlete's heart physiological remodelling: clinical significance, diagnostic strategies and implications for preparticipation screening. Br J Sports Med 2009;43:649-56.

12. Papadakis M, Sharma S. Electrocardiographic screening in athletes: the time is now for universal screening. Br J Sports Med 2009;43:663-8. 